21st Particles and Nuclei International Conference (PANIC 2017)

International Journal of Modern Physics: Conference Series

Vol. 46 (2018) 1860054 (6 pages)

(C) The Author(s)

DOI: $10.1142 / \mathrm{S} 2010194518600546$

\title{
Precision Measurements with an Electroweak Boson in the Final State with the ATLAS Detector
}

\author{
Valerie S. Lang (on behalf of the ATLAS Collaboration) \\ Deutsches Elektronensynchrotron, Notkestr. 85 \\ 22607 Hamburg, Germany \\ valerie.lang@cern.ch
}

Published 3 May 2018

\begin{abstract}
Measurements of the Drell-Yan production of $W$ and $Z / \gamma^{*}$ bosons at the LHC provide a benchmark of our understanding of perturbative QCD and probe the proton structure in a unique way. The ATLAS collaboration has performed new measurements for $W^{+}, W^{-}$ and $Z / \gamma^{*}$ boson production, reaching unprecedented precision and strong constraints on parton distribution functions, in particular the strange quark density. Studying jet production in association with a vector boson, perturbative QCD predictions can be tested in a multi-scale environment. Once the QCD aspects of the vector boson production are understood, high precision measurements aim at the tests of the electroweak sector, such as measuring the $W$ boson mass.

Here, a selection of recent ATLAS measurements, as presented at the 21st Particles and Nuclei International Conference, in September 2017 in Beijing, China, is discussed.
\end{abstract}

Keywords: Electroweak bosons; perturbative QCD; PDFs.

\section{Introduction}

The measurement of $W^{+}, W^{-}$and $Z / \gamma^{*}$ production in proton-proton $(p p)$ scattering at the LHC provides a variety of tests of our understanding of the Standard Model of particle physics. To adequately reproduce the data, good understanding of the electroweak sector and the hard interaction as described by perturbative QCD is needed. Calculations at next-to-leading (NLO) or next-to-next-to-leading order (NNLO) in the strong coupling constant $\alpha_{S}$ and recently at NLO in the electromagnetic coupling $\alpha_{Q E D}$ are required. In addition, the softer contributions around the hard scattering require a profound understanding: the proton content, the amount of radiation before and after the hard interaction as well as the possibility of electroweak radiation from produced quarks. Many of these aspects can be measured in $W$ and $Z$ boson production, and a selection of recent $\operatorname{ATLAS}^{1}$ measurements are discussed in the following.

This is an Open Access article published by World Scientific Publishing Company. It is distributed under the terms of the Creative Commons Attribution 4.0 (CC-BY) License. Further distribution of this work is permitted, provided the original work is properly cited. 


\section{Precision measurements}

The large cross section of $W$ and $Z / \gamma^{*}$ production at the LHC allows measurements of inclusive production cross sections and electroweak parameters with a precision around or below $1 \%$.

\subsection{W boson mass}

The mass of the $W$ boson $\left(m_{W}\right)$ is measured in data of $p p$ collisions ${ }^{2}$, recorded in 2011 at a center-of-mass energy of $7 \mathrm{TeV}$, corresponding to an integrated luminosity of $4.6 \mathrm{fb}^{-1}$. The measurement is performed by optimizing the agreement of the prediction with data under variations of $m_{W}$ in observables which are sensitive to this mass. The challenge lies in the required high precision. The present world average $^{3}$ of $(80385 \pm 15) \mathrm{MeV}$ has an uncertainty of only $0.02 \%$, the value obtained from an electroweak fit ${ }^{4}$ of $(80356 \pm 8) \mathrm{MeV}$ only $0.01 \%$.

Events are selected by requiring the presence of exactly one reconstructed, isolated electron or muon ${ }^{\mathrm{a}}$ with transverse momentum $\left(p_{T}\right)$ above $30 \mathrm{GeV}$ and the signature of the $W$ boson decay neutrino - the missing transverse momentum $\left(\vec{p}_{T}^{\text {miss }}\right)^{\mathrm{b}}$ - to be larger than $30 \mathrm{GeV}$. The transverse mass of the $W$ boson $\left(m_{T}{ }^{c}\right)$ must be larger than $60 \mathrm{GeV}$. For a precise measurement, the $W$ boson should be created almost at rest, hence the reconstructed hadronic recoil has to be below $30 \mathrm{GeV}$. The value of $m_{W}$ is extracted from the $p_{T}^{\ell}$ and the $m_{T}$ distributions, separately for the $W \rightarrow e \nu$ and $W \rightarrow \mu \nu$ decays, for $W^{+}$and $W^{-}$production and 3-4 bins in $|\eta|$.

Fig. 1 (left) shows the measured values for $m_{W}$ from $W^{+}, W^{-}$and $W^{ \pm}$production in comparison with the values from LEP and Tevatron collider experiments. The ATLAS measurement is:

$$
m_{W}=80370 \pm 7 \text { (stat) } \pm 11 \text { (exp. syst) } \pm 14 \text { (model. syst) MeV. }
$$

This is compatible with the world average and of similar precision as the currently leading measurements by the CDF and D0 collaborations ${ }^{5,6}$.

\section{2. $W^{+}, W^{-}$and $Z / \gamma^{*}$ cross sections}

Cross sections of $W^{+}, W^{-}$and $Z / \gamma^{*}$ production are measured in data ${ }^{7}$, recorded at a center-of-mass energy of $7 \mathrm{TeV}$, corresponding to an integrated luminosity of $4.6 \mathrm{fb}^{-1}$. Inclusive cross sections are measured as well as the differential cross section

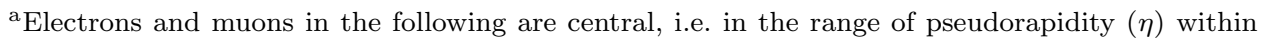
-2.47 to 2.47 ( -2.4 to 2.4 ) for electrons (muons), unless indicated otherwise. For exact numbers see the publication references.

b The $\vec{p}_{T}^{\text {miss }}$ and its absolute value $E_{T}^{\text {miss }}$ are determined from the inverse of the vectorial sum of reconstructed particles and additional soft contributions. In the $W$ mass measurement, the reconstructed hadronic recoil and the lepton transverse momentum are used. For more details, see the respective publication references.

${ }^{\mathrm{c}} m_{T}=\sqrt{2 p_{T}^{\ell} p_{T}^{\text {miss }}\left(1-\cos \Delta \phi\left(\ell, \vec{p}_{T}^{\text {miss }}\right)\right.}$ 
as a function of the lepton pseudorapidity $\left(\left|\eta^{\ell}\right|\right)$ for $W$ production and the dilepton rapidity $\left(\left|y^{\ell \ell}\right|\right)$ in 2-3 bins of the invariant mass $m_{\ell \ell}$ for $Z$ production.

Events are selected by requiring the presence of either exactly one isolated lepton with $p_{T}>25 \mathrm{GeV}$ for $W$ production or two same-flavor, opposite-sign isolated leptons with $p_{T}>20 \mathrm{GeV}$ for $Z$ production. The leptons are required to be central, with one exception. For $m_{\ell \ell}>66 \mathrm{GeV}$, one forward electron is allowed, if the central electron has $p_{T}>23 \mathrm{GeV}$. For $W$ production, events must further have $E_{T}^{\text {miss }}>25 \mathrm{GeV}$ and $m_{T}>40 \mathrm{GeV}$. For $Z$ production, the invariant mass of the dilepton system must be $46(66) \mathrm{GeV}<m_{\ell \ell}<150 \mathrm{GeV}$ (with one forward electron).

Fig. 1 (right) shows the measured inclusive $W$ and $Z$ cross sections vs. NNLO QCD predictions with NLO EW corrections, using six different PDF sets. Apart from the luminosity uncertainty, the inclusive cross section is measured with a precision of $0.5 \%, 0.6 \%$ and $0.32 \%$ for $W^{+}, W^{-}$and $Z / \gamma^{*}$ production. This is contrasted by the difference in the fiducial cross sections between two NNLO QCD calculations, using the same PDF set, of $1.2 \%, 0.7 \%$ and $0.2 \%$, respectively. The experimental precision here challenges the theoretical prediction.

The differential production cross sections for $W$ and $Z$ bosons are used together with the final neutral and charged current deep inelastic scattering data from HERA $\mathrm{I}+\mathrm{II}^{8}$ to extract a new set of PDFs - ATLAS-epWZ16. The result supports the non-suppression of strangeness observed also in the previous ATLAS measurement ${ }^{9}$.

\section{Measurements involving jets}

The measurement of jet production in association with a vector boson probes perturbative QCD predictions in a complementary way to multijet production as a result of the introduced energy scale of the hard interaction of around $(100 \mathrm{GeV})^{2}$.
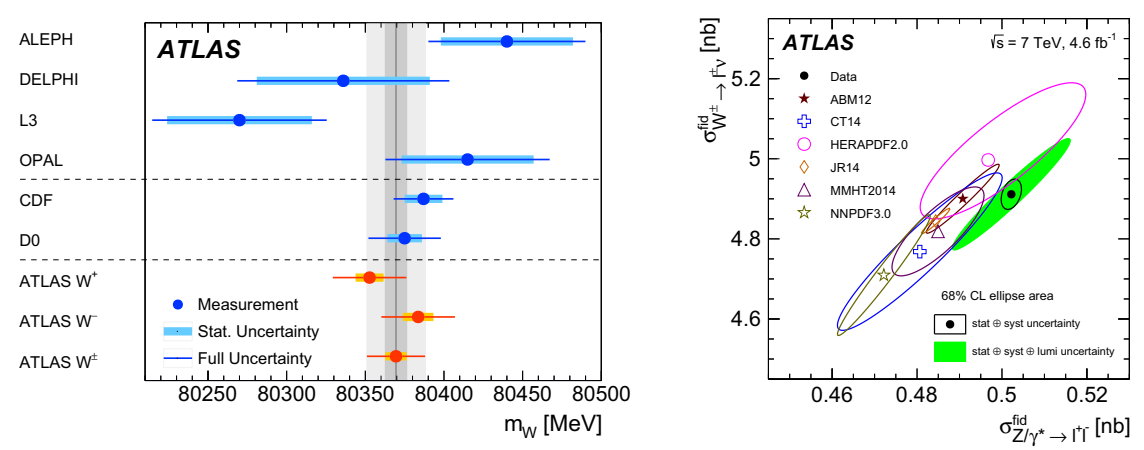

Fig. 1. (Left) The measured value of $m_{W}$ is compared to other published results, including measurements from the LEP experiments ALEPH, DELPHI, L3 and OPAL, and from the Tevatron collider experiments CDF and D0. For more details see Ref. 2. (Right) Integrated fiducial cross sections times leptonic branching ratios of $\sigma_{W^{ \pm} \rightarrow \ell^{ \pm}}^{\mathrm{fid}}$ vs. $\sigma_{Z / \gamma^{*} \rightarrow \ell^{+} \ell^{-}}^{\mathrm{fid}}$. Theoretical predictions based on various PDF sets are shown with open symbols of different colours. For more details see Ref. 7 . 


\section{1. $Z$ boson production in association with jets}

$Z$ boson production in association with up to seven jets is measured in data, recorded in 2015 , with a center-of-mass enery of $13 \mathrm{TeV}$, corresponding to $3.16 \mathrm{fb}^{-1}$ of integrated luminosity ${ }^{10}$. The analysis measures jet multiplicities and multiplicity ratios as well as five differential distributions, all involving jets.

$Z$ boson events are selected in a similar way as in the inclusive cross section measurement at $7 \mathrm{TeV}$ discussed above. The invariant mass window around the $Z$ boson peak is required to be $71 \mathrm{GeV}<m_{\ell \ell}<111 \mathrm{GeV}$. Jets are reconstructed with the anti- $k_{t}$ algorithm ${ }^{11}$ with radius parameter of $R=0.4$. For this analysis, they are required to have $p_{T}>30 \mathrm{GeV}$ and central rapidity, i.e. $|y|<2.5$. Differential distributions are measured as a function of the number of the selected jets.

Fig. 2 (left) shows the measured cross section as a function of $H_{T}$ which is defined as the scalar sum of the transverse momenta of all selected jets and both leptons in the event. This observable is especially sensitive to contributions from higher jet multiplicities. The agreement of the predictions with data improves with the inclusion of an additional jet in the NNLO calculation compared to the NLO BLACKHAT+SHERPA prediction.

\subsection{W boson production in association with jets}

$W$ boson production in association with jets is measured in data of $p p$ collisions at a center-of-mass energy of $8 \mathrm{TeV}$, corresponding to an integrated luminosity of
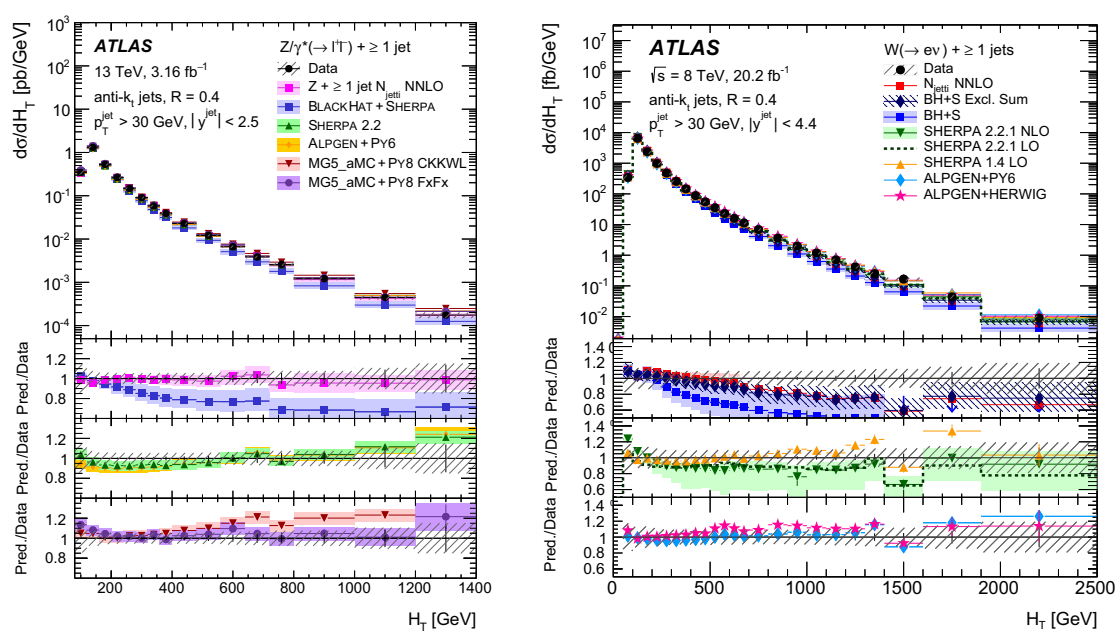

Fig. 2. (Left) Measured cross section as a function of $H_{T}$ for inclusive $Z+\geq 1$ jet events. The data are compared to the predictions from various generators. For more details see Ref. 10. (Right) Cross section for the production of $W$ bosons as a function of the $H_{T}$ for events with $N_{\text {jets }} \geq 1$. For more details see Ref. 12 . 
$20.2 \mathrm{fb}^{-1}$, recorded in $2012^{12}$. Fiducial cross sections as function of the jet multiplicity and eight differential variables test perturbative QCD under various conditions. Measuring $W^{+}$and $W^{-}$production separately, in addition allows to probe valence quark PDFs in a range of the momentum fraction $x$ of the proton which is complementary to inclusive cross section measurements ${ }^{13}$.

Events with $W$ bosons are selected by requiring exactly one isolated electron with $p_{T}>25 \mathrm{GeV}, E_{T}^{\text {miss }}>25 \mathrm{GeV}$ and $m_{T}>40 \mathrm{GeV}$. Events with a central jet with $p_{T}>20 \mathrm{GeV}$ being tagged as containing a $b$-hadron are rejected, using a $b$-jet tagging algorithm with $60 \%$ efficiency. This suppresses $t \bar{t}$ production - the dominant background at high jet multiplicities. Signal jets are selected in this analysis similar to the $Z+$ jets analysis discussed above, but including rapidities up to 4.4.

Fig. 2 (right) displays the $H_{T}$ distribution for $W$ boson production in association with at least one jet. Similar to $Z+\geq 1$ jet production, the NNLO calculation of $W+\geq 1$ jet production improves clearly on the description of data compared to the NLO prediction by BLACKHAT+SHERPA. The exclusive sums approach in BLACKHAT+SHERPA achieves a similar agreement with data as NNLO. In both cases, the description of the $H_{T}$ distribution in data is worse under the inclusion of forward jets, compared to $Z+\geq 1$ jet in Fig. 2 (left).

Fig. 3 (left) displays the measured ratio of $W^{+}$to $W^{-}$production as a function of the $W$ boson $p_{T}$. The NLO prediction by MCFM is shown with four different PDF sets. The choice of PDF sets modifies the prediction of the $W^{+}$to $W^{-}$cross section ratio by around the experimental uncertainty in part of the covered $W p_{T}$ range. The data will therefore be very interesting as input to future fits of PDFs.
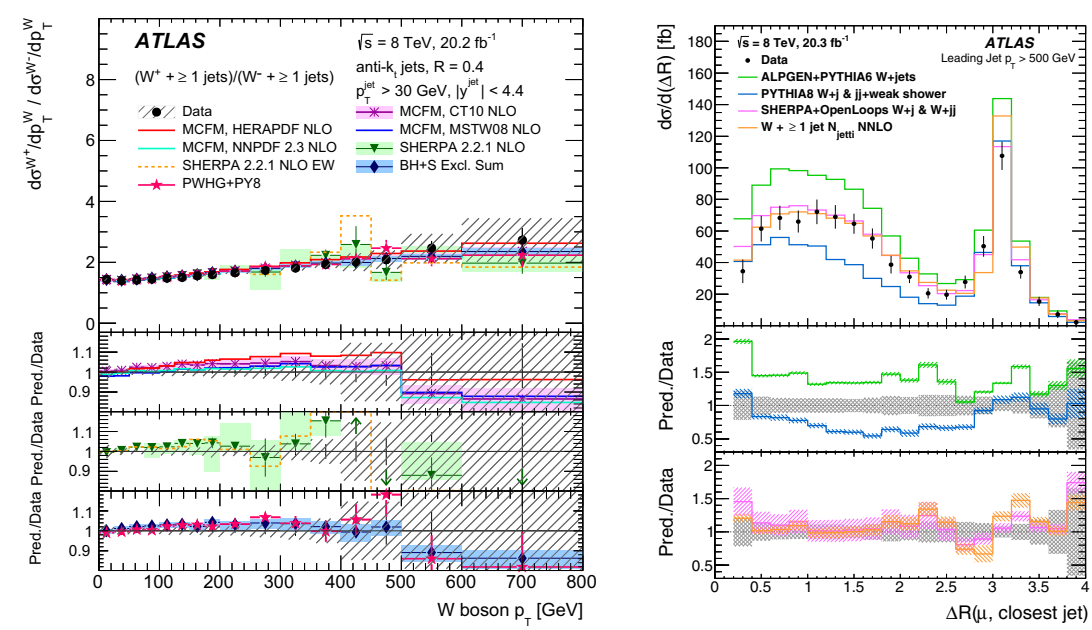

Fig. 3. (Left) $W^{+}$to $W^{-}$cross-section ratio as a function $W p_{T}$ for events with $N_{\text {jets }} \geq 1$. For more details see Ref. 12. (Right) Unfolded distribution from background-subtracted data of the angular separation between the muon and the closest jet in the signal region along with several predictions from theory calculations. For more details see Ref. 14 . 


\section{Specialized measurements}

Measurements of $W$ and $Z$ boson production in special corners of phase space test theoretical predictions in particular detail, often close to divergencies.

\subsection{Collinear $W$ boson emission in dijet topologies}

The angular distribution of $W$ boson emission is measured in events with high transverse momentum jets in data of $p p$ collisions ${ }^{14}$ at a center-of-mass energy of $8 \mathrm{TeV}$, corresponding to an integrated luminosity of $20.3 \mathrm{fb}^{-1}$.

Events are selected by requiring the presence of exactly one muon with $p_{T}>$ $25 \mathrm{GeV}$, no $b$-tagged jet (using a tagging algorithm with $70 \%$ efficiency) and at least one jet with $p_{T}>500 \mathrm{GeV}$. Only central jets $(|\eta|<2.1)$ are considered. The angular distance $\Delta R$ of the muon is measured with respect to the closest jet with $p_{T}>100 \mathrm{GeV}$.

Fig. 3 (right) displays the angular separation $\Delta R(\mu$, closest jet). For close muonjet separations $(\Delta R<2.4)$ clearly NLO QCD calculations including NLO EW corrections or NNLO QCD calculations are required to describe the data. The amount of collinear $W$ boson radiation is found to increase with higher leading jet $p_{T}$.

\section{Conclusions}

A variety of interesting measurements of $W$ and $Z$ boson production have been performed by the ATLAS Collaboration at the LHC with data from $p p$ collisions at center-of-mass energies of 7,8 and $13 \mathrm{TeV}$. These measurements probe various aspects of our understanding of the Standard Model of particle physics. More data already recorded and to be recorded in the next years - will provide further insights.

\section{References}

1. ATLAS Collab., JINST 3, S08003 (2008).

2. ATLAS Collab., arXiv:1701.07240 [hep-ex] (2017).

3. Particle Data Group (C. Patrignani et al.), Chin. Phys. C, 40, 100001 (2016) and 2017 update.

4. Gfitter Group (M. Baak et al.), Eur. Phys. J. C74, 3046 (2014).

5. CDF Collab., Phys. Rev. Lett., 108, 151803 (2012).

6. D0 Collab., Phys. Rev. Lett., 108, 151804 (2012).

7. ATLAS Collab., Eur. Phys. J. C77, 367 (2017).

8. ZEUS+H1 Collab., Eur. Phys. J. C75, 580 (2015).

9. ATLAS Collab., Phys. Rev. Lett. 109, 012001 (2012).

10. ATLAS Collab., Eur. Phys. J. C77, 361 (2017).

11. M. Cacciari, G. P. Salam and G. Soyez, JHEP 04, 063 (2008).

12. ATLAS Collab., arXiv:1711.03296 [hep-ex] (2017).

13. S. A. Malik and G. Watt, JHEP 02, 025 (2014).

14. ATLAS Collab., Phys. Lett. B765 132-153 (2017). 